\title{
SPECTROSCOPIC INVESTIGATIONS OF HALOS OF PLANETARY NEBULAE
}

\author{
M.BäSSGEN ${ }^{*}$, G.BÄSSGEN ${ }^{*}$, M.GREWING ${ }^{*}$, S.CERRATO ${ }^{*}$, L.BIANCHI ${ }^{* *}$ \\ * Astronomisches Institut Tübingen (FRG) \\ Osservatorio Astronomico di Torino (Italy)
}

Different ESO-telescopes with medium resolution spectrographs and CCD-detectors were used to look for emission from extended halos around Planetary Nebulae. In three out of four cases the search was successful. The ratio $R_{h a l o} / R_{\text {neb }}$ ranges from 2 to about 10 .

Assuming that the halos have been produced by the winds of the Red Giant progenitor stars, we determine $\dot{M} / V_{A G B}$ of these progenitors, using the measured $H \alpha-$ and $H B$ fluxes. These values scatter in a relatively narrow range around $2 . .5 \cdot 10^{-7}(\mathrm{Mo} / \mathrm{y}) /(\mathrm{km} / \mathrm{s})$. In some objects emission line fluxes were measured at two ore more radial distances and radial density profiles were derived from them. These are in good agreement with the expected $n \sim r^{-2}$ density law expected for a freely expanding wind.

Extrapolating the halo density profile inwards we can determine $n_{n e b} / n_{\text {halo }}$ at the location of the nebula. With one exception, this ratio is larger than 4 , indicating that the compression of the gas was caused by an isothermal shock rather than by an adiabatic shock.

The derived $\dot{M} / \mathrm{V}$ values, combined with known mass loss rates and wind velocities of the central stars, are used to test the two-wind model (Kwok et al. 1978) which describes a possible formation scenario of planetary nebulae.

The conclusion seems unavoidable that in the very late stage of the evolution of the progenitor star an increased mass loss rate is required to explain the observed nebular properties.

$\dot{\mathrm{M}} / \mathrm{V}$ calculated from emission line fluxes in units of $10^{-7}(\mathrm{Mo} / \mathrm{y}) /(\mathrm{km} / \mathrm{s})$

\begin{tabular}{|c|c|c|}
\hline Obj. & Pos. & $\dot{\mathbf{M}} / \mathbf{V}$ \\
\hline NGC 1535 & $\begin{array}{l}31^{\prime \prime} \\
40^{\prime \prime}\end{array}$ & $\begin{array}{l}3.0 \\
3.5\end{array}$ \\
\hline NGC 2452 & $\begin{array}{l}11^{\prime \prime} \\
17^{\prime \prime}\end{array}$ & $\begin{array}{l}17.0 \\
11.0\end{array}$ \\
\hline NGC 2792 & $17^{\prime \prime}$ & 2.5 \\
\hline NGC 2867 & $\begin{array}{l}17^{\prime \prime} \\
34^{\prime \prime}\end{array}$ & $\begin{array}{l}1.8 \\
1.8\end{array}$ \\
\hline NGC 3132 & $\begin{array}{l}30^{\prime \prime} \\
54^{\prime \prime}\end{array}$ & $\begin{array}{l}7.0 \\
4.5\end{array}$ \\
\hline NGC 3211 & $19^{\prime \prime}$ & 2.2 \\
\hline
\end{tabular}

\begin{tabular}{llr} 
Obj. & Pos. & \multicolumn{1}{c}{ M/V } \\
\hline NGC 3918 & $25^{\prime \prime}$ & 2.2 \\
IC 418 & $15^{\prime \prime}$ & 8.0 \\
& 29 & 6.0 \\
IC 2165 & $10^{\prime \prime}$ & 3.5 \\
& $17^{\prime \prime}$ & 3.3 \\
& $27^{\prime \prime}$ & 4.0 \\
IC 2448 & $17^{\prime \prime}$ & 2.5 \\
& $29^{\prime \prime}$ & $2.0:$ \\
IC 2501 & $20^{\prime \prime}$ & 2.0
\end{tabular}

S. Torres-Peimbert (ed.), Planetary Nebulae, 199.

(c) 1989 by the IAU. 